\title{
基于热场域分割的体参数化模型构建
}

\author{
陈龙，伯龙飞，卜宁远，汪中厚 \\ (上海理工大学机械工程学院 上海 200093) \\ (c1@usst.edu.cn)
}

\begin{abstract}
摘 要: 针对当前拓扑优化结果需要后处理的问题, 提出一种适用于有限元四边形网格和六面体网格的体参数化模 型构建方法. 首先根据热场特点, 在有限元网格上施加稳态热场并通过有限元求解得到热场域数据; 然后针对热场 域值做一系列子域分割操作, 包括抽取几何表面网格、等值域提取、邻域去重叠、角点与交点求取和非四边形域划 分, 得到全部的四边形子域; 最后对所有四边形子域离散边界做点排序及 $\mathrm{B}$ 样条控制点的反求, 再利用子域插值算 法实现复杂几何模型体参数化表达. 给出的二维和三维实例结果表明, 该方法能准确、高效地实现复杂模型的子域分 割，进而构建适用于等几何分析的体参数化模型，从而为拓扑优化后处理提供一个可行途径.
\end{abstract}

关键词：体参数化; 热场; 有限元网格; 域分割; 拓扑优化

中图法分类号: TP391.41 DOI: 10.3724/SP.J.1089.2020.18266

\section{Construction of Volume Parametric Model Based on Segmentation of Thermal Field}

Chen Long, Bo Longfei, Bu Ningyuan, and Wang Zhonghou

(College of Mechanical Engineering, University of Shanghai for Science and Technology, Shanghai 200093)

\begin{abstract}
In term of the post-processing of topology, a construction algorithm is proposed for the volumetric parameterized model which is suitable for the finite element quadrilateral meshes and the hexahedral meshes. Firstly, a steady thermal field is applied to the finite element mesh model and the thermal field data is obtained by the finite element method according to the characteristics of thermal field. Then, a series of sub-domain segmentation operations are performed on the thermal field data, such as extracting geometric surface mesh, extracting equal-value domain, removing neighborhood overlap, finding corner point and contour line intersection on geometric boundary and dividing non-quadrilateral domain, so as to obtain all the quadrilateral sub-domains. In the end, sorting point and obtaining the control points of B splines are performed for the discrete boundary of the quadrilateral subdomains. The volume interpolation algorithm is used to realize the volume parameterization construction of complex model. The example results of $2 \mathrm{D}$ and $3 \mathrm{D}$ show that the algorithm can accurately and efficiently realize the subdomain segmentation and the volume parameterization construction of complex models that are suitable for isogeometry analysis. Our method provides a feasible approach for the post-processing of topology optimization.
\end{abstract}

Key words: volume parameterization; thermal field; finite element meshes; sub-domain segmentation; topology optimization

收稿日期：2020-03-07; 修回日期：2020-08-10. 基金项目：国家自然科学基金(52075340，51475309，51875360). 陈龙(1978一), 男, 博士, 副教授, 硕士生导师, CCF 高级会员, 主要研究方向为产品智能计算设计、机器视觉与机器人; 伯龙飞(1993一), 男, 硕士 研究生, 主要研究方向为 CAD; 卜宁远(1997一), 男, 硕士研究生, 主要研究方向为 CAD; 汪中厚(1963一), 男, 博士, 教授, 博士生 导师, 主要研究方向为机械传动与动力学、有限元技术及应用. 
通常拓扑优化结果是用有限元网格表达的模 型, 将拓扑优化结果转化为 STL 格式是常见的一 种操作, 该格式目前被三维打印机和 CAD 软件广 泛认可 ${ }^{[1-2]}$; 然而三维打印因增加大量几何支撑点 会使得几何表面质量很差 ${ }^{[3]}$. 因此, 如果在尺寸、 形状和表面光饰度方面有严格要求，则需要进一 步对拓扑优化进行后处理。

不仅如此，在拓扑优化驱动设计和制造创新 中, 将离散有限元网格模型表达为连续的边界表 达模型或参数化模型具有更重要的意义 ${ }^{[4]}$. (1) 离 散有限元网格模型无法和已有的 CAD 格式兼容, 必须要转化为 $\mathrm{CAD}$ 模型固有格式如边界表达模 型,且能生成参数化的、可编辑的模型. (2) 基于边 界表达的几何模型，不仅可以清晰地表示边界，而 且可以嵌人更高阶的几何信息，如法向量、曲率等， 这些信息不仅可以用来辅助产品的生产制造，而 且用于设计和制造过程之间建立无缝连接 ${ }^{[4-5]}$.

将拓扑优化有限元网格模型转化为边界表达 的参数化模型，不仅能够精确地表示光滑的物体 表面 ${ }^{[6]}$, 且有利于计算机进行曲面的存储、分析、 计算和可视化绘制. 基于参数化的模型基础, 利用 参数化设计能大幅度减少底层建模的工作，目前 已经有大量研究 ${ }^{[7-9]}$. 将有限元网格模型转化为体 参数化模型, 则是近年来兴起的一种新方法. 体参 数化模型不仅具有边界参数化模型的优势, 而且 能提供模型体内各部分的非均质材料连续性表示, 使得体参数化模型比传统的 B-Rep ${ }^{[10]}$ 和 $\mathrm{CSG}^{[11]}$ 在 非均质模型表达和设计方面更具有优势. 同时，基 于 B 样条和 NURBS 样条等表达的体参数化模型可 以直接进行有限元分析和等几何分析，不必对其 再进行网格划分，从而实现 CAD 与 CAE 之间的无 缝集成 ${ }^{[12]}$.

本文面向拓扑优化或有限元分析结果的有限 元网格模型，提出一种构建体参数化模型的方法. 针对有限元四边形和六面体网格, 利用有限元分 析得到该模型的热场信息，并利用一系列操作实 现子域分割，得到多个四边形子域或六面体子域, 最后利用体参数化模型构建方法得到能用于等几 何分析的体参数化模型, 从而完成拓扑优化结果 的后处理.

\section{1 相关工作}

\section{1 域分割方法}

$\mathrm{B}$ 样条体参数化模型构建前, 需要将复杂模型
划分为四边形或六面体子域，这个操作类似于传 统有限元网格生成中的子域生成. 一直以来, 有限 元中子域生成采用手动切割实体来实现，而最近 等几何分析技术的兴起也亟需解决手动分割带来 的弊端. 四边形网格自动域划分的方法, 更多的是 以三角剖分为底层网格，在此基础上的网格合并 算法可实现几何图形划分成大的四边形域，如胡 向红等 ${ }^{[13]}$ 提出由区域生长算法实现域的四边形划 分. 对于 $N(N \neq 4)$ 边几何分割, 孙春华等 ${ }^{[14]}$ 曾使用 Gregory 算法 ${ }^{[15]}$, 通过共轭梯度数值为优化函数解 决了 $N$ 边域的自动划分, 并生成参数化的非自交 网格. 李毅等 ${ }^{[16]}$ 以 Q-Morph 算法 ${ }^{[17]}$ 为基础, 实现 了增加约束后自动生成二维多边形域的划分. 赵 国群等 ${ }^{[18]}$ 在栅格法的基础上，提出表层网格法实 现六面体网格自动划分与重划分. 陈龙等 ${ }^{[12]}$ 利用 一系列网格操作实现了四边形网格模型的子域分 割, 并进而将四边形网格模型重建为体参数化模 型，该方法能处理任意复杂的二维模型，但是其较 多地依赖一些经验参数.

\section{2 体参数化}

体参数化几何的构建是等几何分析的第 1 步, 根据空间数据分布为二变量和三变量张量体, 一 般有创建式和重建式 2 种方式 ${ }^{[19-20]}$. Martin 等 ${ }^{[21]}$ 提 出了利用体积调和函数将三角形网格模型拟合成 $\mathrm{B}$ 样条体的方法, 然而其对 B 样条很难实现封闭圆 或者椭圆的几何模型，因此引入了 NURBS 曲线曲 面. Aigner 等 ${ }^{[22]}$ 提出一种构造扫掠体 NURBS 体参 数化变分方法. 重建法生成体参数化模型的原理是 从点云模型、多边形网格模型、标准格式(B-Rep)模 型和 CSG 等已有模型出发进行模型参数化重建 ${ }^{[23-24]}$.

\section{2 方法概述}

几何域添加物理矢量场后, 根据矢量场来指导 几何域分割, 是一个重要的方法 ${ }^{[25-26]}$. 物理场包括 热场、力场、电磁场和位移场等, 相对于应力和应 变等向量场来说, 温度场是一个标量场, 使用标量 场能够简化矢量场的复杂程度. 由于第 1 类边界条 件下的热传导是线性的，从而保证域分割具有均匀 性和整体对称性，可避免域内边界产生自交现象和 减少域的奇异性，且所有等值线将垂直于几何边 界. 因此关于域分割可转化为求解稳态热场的问 题，并且只需满足第 1 类边界条件 $\left.\boldsymbol{T}\right|_{\Gamma}=\boldsymbol{T}_{w}$. 其中, $\Gamma$ 为物体边界; $\boldsymbol{T}_{w}$ 为已知边界温度 ${ }^{[27]}$, 单位为 ${ }^{\circ} \mathrm{C}$. 
第 1 类边界条件是几何边界上的函数, 这里 $\left.\boldsymbol{T}\right|_{\Gamma}$ 为温度, 表示为

$$
\left\{\begin{array}{l}
\left.\boldsymbol{T}\right|_{\Gamma}=\boldsymbol{T}_{w} \\
\left.\boldsymbol{T}\right|_{\Gamma}=f(x, y, t)
\end{array}\right.
$$

其中, $f(x, y, t)$ 为边界温度函数. 为了保证温度的 合理分布，边界极限取值范围为 $0 \leqslant T \leqslant 1$.

本文以有限元原始网格为背景网格，通过有
限元法求解出每个节点温度场值. 在给定的角点 约束抽取等值网格面, 并对邻域施加去重叠操作, 求解域边界交点与几何角点, 根据多边形划分规 则对非四边形域进行再次分割. 对边界点进行排 序，利用最小二乘法实现 B 样条曲线拟合，再利用 Coons 插值得到内部控制点, 进而得到体参数化模 型. 具体流程如图 1 所示.

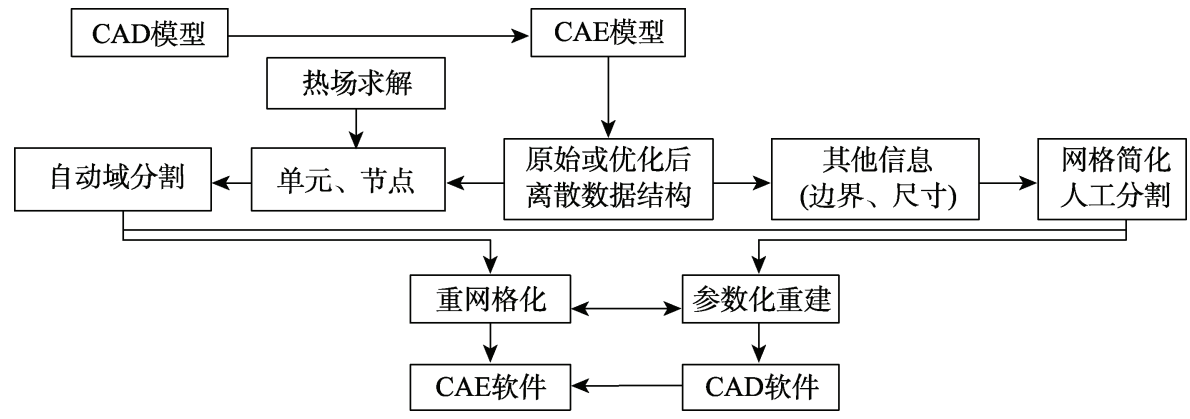

图 1 本文方法流程

\section{3 稳态热场有限元求解及预处理}

稳态温度场的求解是一个非常成熟的技术， 采用有限元软件进行求解，步骤如下.

输人. CAD 模型.

输出. CAE 几何域的热场求解结果.

Step1. 设置几何材料属性、热传导系数和单元类型.

Step2. 施加边界条件. 图 2a 所示为 CAD 模型, 图 $2 \mathrm{~b}$ 所示为对模型施加第 1 类边界条件. 为保证几何的对 称性, 温度值施加在模型的局部对称中心的节点上或者 模型两端节点上.

Step3. 模型求解, 稳态热解如图 2c 所示.

Step4. 提取几何信息，包括几何模型节点(NLIST), 单元(ELIST)及节点温度值(TLIST).

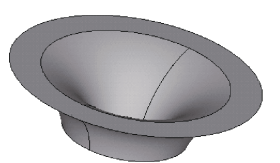

a. $\mathrm{CAD}$ 模型

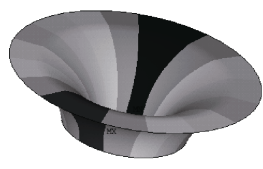

c. 求解结果

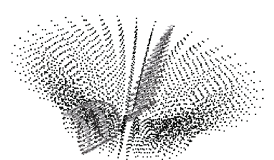

b. 施加边界条件

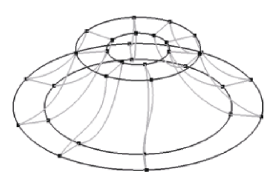

d.等温线
图 2 温度场与等温线

\section{4 模型域分割}

已有有限元节点 $N$ 和单元 $E$, 由于并不是所有
四边形网格或六面体网格都为 4 节点四边形网格 或 8 节点六面体网格, 对它们进行后续处理比较困 难. 因此, 将非 4 节点四边形网格转化为 4 节点四 边形单元网格, 非 8 节点六面体网格转化为 8 节点 六面体网格, 转化过程如图 3 所示.
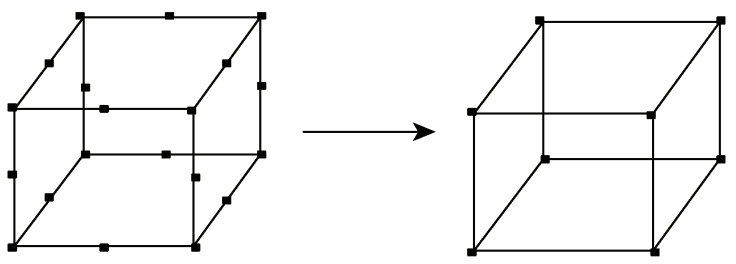

图 3 六面体网格节点简化

根据温度场对几何域进行分割, 主要步骤包 括：离散网格表面提取、抽取等值网格表面、去 除邻域重叠、求取几何边界角点和域边界交点、 四边形域剖分, 流程如图 4 所示.

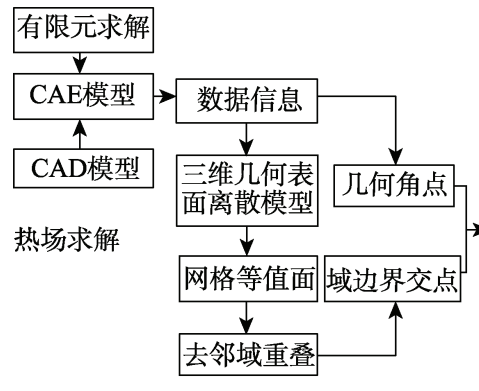

B样条体 参数化模型 线性插值 内部控制点 判断域性质 分割非四边 反求子域控 反制点与拟合 \begin{tabular}{c} 
分割非域 \\
\hline
\end{tabular} 排序子域 四边形子 模型边界 划分四边形子域 B样条体参数化

图 4 域分割方法 


\section{1 域分割方法}

如何处理离散的六面体网格几何信息，主要 有 3 种方式: (1) 将六面体单元作为对象进行合并 处理，容易出现域边界不连续现象；(2) 以所有的 边为对象进行合并处理，容易出现子域不连续或 者子域不封闭现象; (3) 以六面体 6 个表面为对象. 其中第 3 种方式具有较好效果. 提取单元所有的表 面后，由于相邻单元之间一定存在重合面，因此剔 除所有重合面, 剩下的面即为边界表面. 当提取三 维模型所有的边界面后, 三维模型即转化为曲面 网格模型, 如图 5 所示.

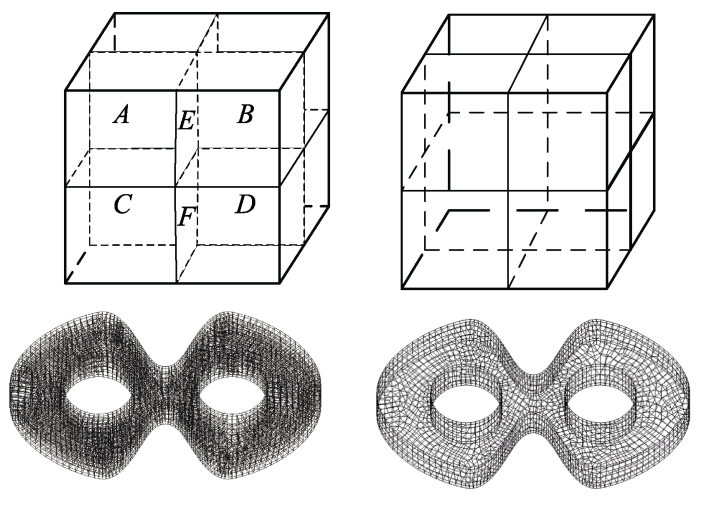

图 5 表面网格提取

\section{2 获取等值子域网格}

以四边形网格为对象，通过设置合理阈值，即 可实现对等温网格面的分割. 在分割过程中，子域 边界多为锯齿形状, 且会出现邻域重叠现象, 但是 并不影响后处理的结果. 原始网格越稀疏，则锯齿 状越明显、域划分越粗糙、越会影响后面子域的整 体质量以及体参数化结果. 但是, 过密的网格会大 幅度降低计算效率。

\section{3 去除邻域网格重叠}

（1）重叠现象的原因. 由于网格是四边形，网 格顶点温度场值不同, 当四边形网格 4 个节点至少 有 3 个节点温度场值属于该阈值时，子域之间会出 现不连续现象，这会使得后期处理非常困难. 为保 证域之间连续性, 若四边形网格中至少有 2 个节点 温度场值属于该阈值，则判断其为该子域等值网 格，因此抽取等值网格邻域之间会出现重叠现象.

（2）重叠约束条件. 至少 2 个子域相邻, 四边形 网格 4 个节点至少有 2 个节点温度场值属于该阈值.

去重叠方法如图 6 所示. 图 6a 设置初始域四 边形单元集合 $S_{1}$; 设置与其域相交的邻域四边形 单元集合 $S_{2}$; 灰色部分为 $S_{1}$ 与 $S_{2}$ 重叠部分; 当 $S_{1}$ 里的单元等于 $S_{2}$ 里的单元时, 剔除 $S_{2}$ 里相同的四
边形单元; 这样得到一个 $S$ 如图 $6 \mathrm{~b}$ 所示新四边形 单元集合; 然后迭代所有邻域, 并全部去除子域的 重叠现象.
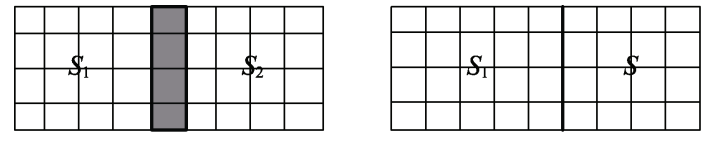

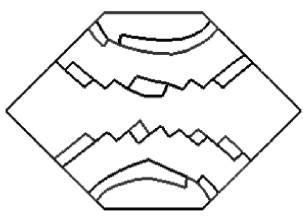

a. 去重叠前

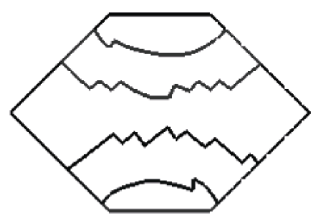

b. 去重叠后
图 6 去重叠示意图

\section{4 求模型角点及子域交点}

为了判断子域的性质及其对离散边界进行排 序拟合, 需要获取模型及其子域的边界及其边界 交点. 首先将子域边界分为 2 类: 一类是几何模型 边界与子域边界重合部分(下文统称为几何域边 界); 另一类是不重合部分，称为等值线.

\subsection{1 获取几何子域边界}

输人. 几何子域的单元网格.

输出. 子域边界.

Step1. 以单元网格为对象, 获取所有四边形或六 面体网格的离散边. 获取几何模型的边界时, 以整个模 型单元的网格作为输人; 获取子域边界时, 以单个子域 所有的网格作为输人.

Step2. 如果一条离散边的 2 个顶点与另一条离散 边的 2 个顶点重合, 则判断为几何模型的内部离散边; 否则为模型边界的离散边 $A$.

Step3. 依据 Step2 方法, 再获取去重叠后的子域边 界边 $B$

Step4. 查找 $A$ 与 $B$ 相等的部分, $A \cap B$ 为几何子域 边界边, $B-(A \cap B)$ 则为等温线.

\subsection{2 获取几何角点}

图 $7 \mathrm{a}$ 和图 $7 \mathrm{~b}$ 是角点存在的 2 种形式, 根据边 界向量夹角阈值 $\alpha$ 来判断此点是否为角点. 已知 提取所有几何离散边界, 离散边界中以任意离散 边 $\left(\boldsymbol{X}_{0}, \boldsymbol{Y}_{0}\right)$ 的节点 $\left(x_{0}, y_{0}\right)$ 为起始位置, 寻找其离散 边的第 2 个节点 $\left(x_{1}, y_{1}\right)$ 相邻的边 $\left(\boldsymbol{X}_{1}, \boldsymbol{Y}_{1}\right)$, 求出 2 条相邻边向量夹角 $\theta$ 值，当 $\theta \geqslant \alpha$ 时，则为边界角 点. 其中,

$$
\cos (\theta)=\frac{\left(\boldsymbol{X}_{i}, \boldsymbol{Y}_{i}\right) \times\left(\boldsymbol{X}_{i+1}, \boldsymbol{Y}_{i+1}\right)}{\left|\left(\boldsymbol{X}_{i}, \boldsymbol{Y}_{i}\right)\right|\left(\boldsymbol{X}_{i+1}, \boldsymbol{Y}_{i+1}\right) \mid}
$$

图 7c 所示为六边形未求取几何角点时拟合几 


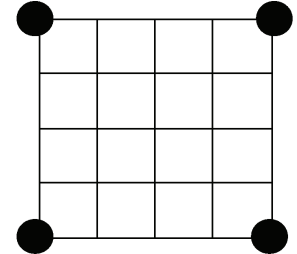

a. 角点存在一个单元上

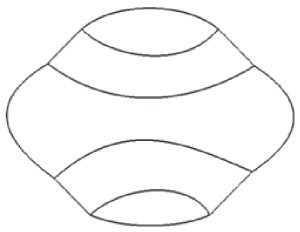

c. 获取角点之前边界拟合

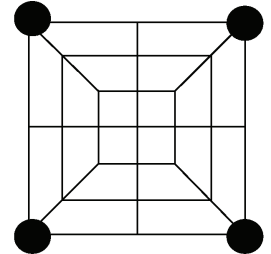

b. 角点存在多个单元上

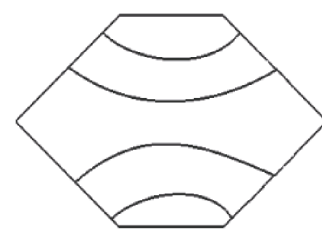

d. 获取角点之后边界拟合
图 7 获取几何角点

何边界, 显然与原始六边形模型出现失真情况; 图 $7 \mathrm{~d}$ 所示为本文方法获取图 $7 \mathrm{~d}$ 类型的角点方式之后 拟合几何边界，结果显示该方法可以处理任意复 杂的情况，可恢复原始几何边界模型.

\subsection{3 获取域边界交点}

上述方法用来获取离散几何域的边界和等值 线. 2 种边界都是以单个子域为对象来存储, 以方 便对每个子域边界进行求交. 2 种边界可以任何一 种边界来获取子域的边界交点, 此时只需对一条 边界所有离散边的节点集合进行比较，若某节点 仅出现一次, 则该节点为此边界的端点即子域边 界之间的交点.

\section{5 非四边形子域划分}

上述算法并不能完全实现模型划分为四边形 子域，因此需要进一步对非四边形子域进行划分.

Step1. 判断子域奇偶性. 根据模型角点数量 $N$ 与 子域边界交点数量 $M$ 之和判断每一个子域是否为四边 形子域; 当 $N+M=4$ 时, 则其为四边形子域; 当 $N+M \neq 4 \cup(N+M) \% 2=0$ 时，则其为偶数边域，否 则为奇数边子域. 如图 8 所示, 圆形表示几何角点, 正 方形表示子域边界交点, 子域 $A, C, D, E$ 中 $N+M=4$ 则 为四边形子域; 子域 $B$ 为偶数边子域.

Step2. 偶数多边形子域划分, 如图 9 所示. 前沿边 的确定, 生长侧边, 连接侧边端点生成顶边, 保证 $f$ 值 最小. 该算法只对边界敏感, 建立约束条件: 以奇异边 界交点(边界内角大于 $135^{\circ}$ ) 为待分割点; 边界在子域内 部严格不相交; 以域的角度 $\theta=a \cos (k)$ 标准差最小值 来确定待分割子域. 利用向量之间的关系得出目标函数

$$
f=\frac{1}{4} \sum_{i=0}^{3}\left|a \cos \left(k_{i}\right)-\frac{1}{4} \sum_{i=0}^{3} a \cos \left(k_{i}\right)\right|
$$

Step3. 奇数多边形子域划分, 如图 10 所示. 已知
一个奇数 $N$ 边形子域, 各顶点为 $\boldsymbol{V}_{k} ; O$ 为其多边形域 的中心点; 第 $k$ 条边记为 $\boldsymbol{E}_{k}=(1-s) \boldsymbol{V}_{k}+s \boldsymbol{V}_{k+1} ; \boldsymbol{E}_{k}$ 中 点 $\boldsymbol{M}_{k}=0.5\left(\boldsymbol{V}_{k}+\boldsymbol{V}_{k+1}\right)$. 由此在参数域内构成了 $n$ 个由 $\boldsymbol{O} \boldsymbol{M}_{k} \boldsymbol{V}_{k} \boldsymbol{M}_{k-1}$ 组成的四边形.

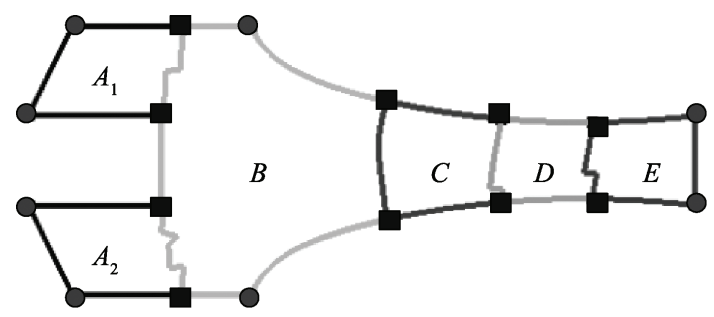

图 8 子域性质
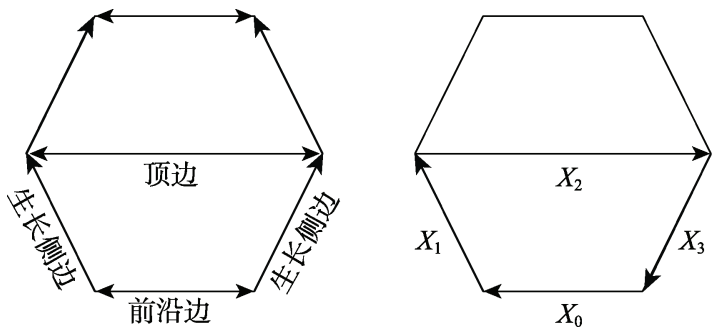

图 9 偶数边子域划分

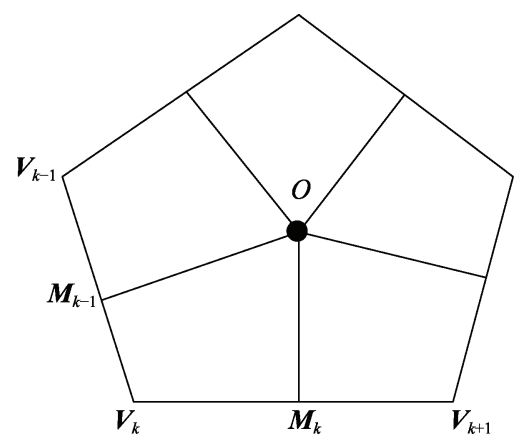

图 10 奇数边子域划分

\section{5 体参数化模型生成}

\section{1 去除邻域网格重叠}

由于域边界多为锯齿形，需要对离散几何边 界进行优化拟合，且 $\mathrm{B}$ 样条曲线曲面是具有方向 性张量积性质的参数化曲线曲面, 因此每一条待 优化的边界曲线都具有一定的方向, 此时应该对 离散边进行排序.

首先确定一个起始子域，一般把等温值最小 或最大的子域作为起始子域; 然后分别对子域的 每条离散边进行排序. 具体步骤如图 11 所示, 获 取起始边与起始点后, 把起始边放到存储容器的 首地址, 并寻找其邻边, 迭代此过程, 直到遇到具 有交点的离散边结束一条边的排序. 图 11 是一条 
边排序的算法流程图, 整个几何域边界排序只需 进行有限次的迭代运算，并且相同温度域不同区 域情况分开处理. 处理完成后, 此时剖分的结果只 适用于二维单个子域，对于三维等温域之间拼接 处局部求得交点不重合，导致域不连续，如图 12 所示. 要想让 2 个子域连续, 方形点必须是同一位 置. 为了解决这一问题，只需在求得域之间交点时 把第 1 个子域的交点作为基础域, 遍历待求邻域的 离散边界节点; 当第 1 个子域交点等于邻域边界节 点时, 此节点为邻域的交点, 以此来解决局部交点 不重合现象.

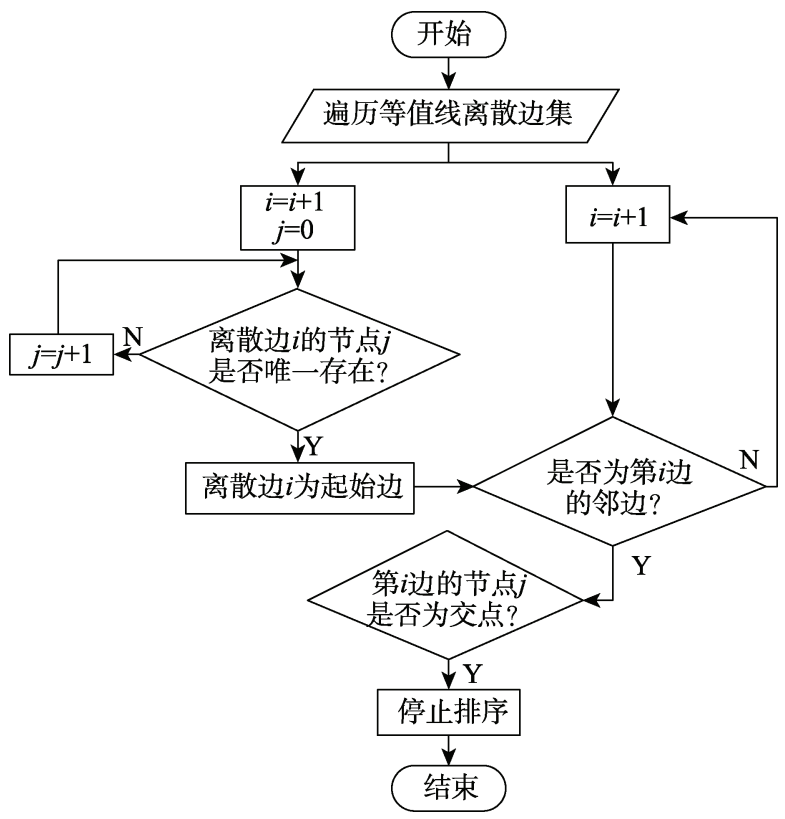

图 11 边界排序算法流程图
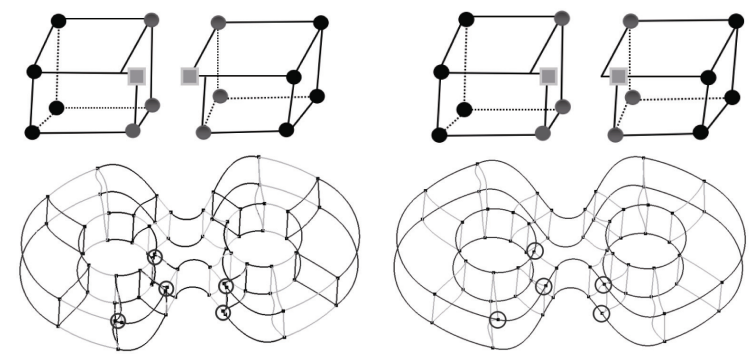

图 12 域之间交点不重合情形

\section{2 体参数化模型插值生成}

由于几何边界为未知曲线或直线，为了更好 地表达几何边界和逼近原始几何模型，采用最小 二乘法 $\mathrm{B}$ 样条曲线拟合几何离散边界 ${ }^{[28-29]}$, 并反 求出边界控制点. 取 $f$ 极小值, 如

$$
f=\sum_{k=1}^{r-1}\left|\boldsymbol{Q}_{k}-\boldsymbol{C}\left(\overline{\boldsymbol{u}}_{k}\right)\right|^{2}
$$

其中, $\boldsymbol{Q}_{k}$ 为待拟合离散节点; $\boldsymbol{C}\left(\overline{\boldsymbol{u}}_{k}\right)$ 为 $\mathrm{B}$ 样条曲 线表达式，使得曲线 $\boldsymbol{C}\left(\overline{\boldsymbol{u}}_{k}\right)$ 无限逼近离散边界.
其次，根据 Coons 曲面插值算法 ${ }^{[30-31]}$ 推导出 三维实体插值算法, 得出其内部控制点 $\boldsymbol{P}_{i, j, k}$. 在 反求控制点时, 定义曲线为 3 次 4 阶的 4 个控制点 $\mathrm{B}$ 样条曲线, 以此可设体参数化每个子域的控制点 为 64 个. $u, v, \omega$ 方向次数均为 3 次, 节点向量 $\boldsymbol{u}=\boldsymbol{v}=\boldsymbol{\omega}=\{0,0,0,0,1,1,1,1\}$, 实现 B 样体参数化表 达, 即

$$
\begin{gathered}
\boldsymbol{V}(\boldsymbol{u}, \boldsymbol{v}, \boldsymbol{\omega})=\sum_{i=0}^{n} \sum_{j=0}^{m} \sum_{k=0}^{l} \boldsymbol{N}_{i, p}(\boldsymbol{u})_{j, q} \times \boldsymbol{N}(\boldsymbol{v}) \times \\
\boldsymbol{N}_{k, r}(\boldsymbol{\omega}) \times \boldsymbol{P}_{i, j, k}(x, y, z)
\end{gathered}
$$

\section{6 算法与实例}

根据第 5 节算法可以很容易地实现二维几何 域的划分及其参数化表达; 同时在二维的基础上 对该算法进行强化, 很容易从二维域映射到三维 几何. 本文以拓扑优化后的网格为对象, 表 1 所示 为图 13 所示 2 个模型的基础数据, 采用第 5 节算 法, 实现 B 样条体参数化.

表 1 模型数据

\begin{tabular}{crrccc}
\hline 模型 & 节点数 & 单元数 & 子域数 & 交点数 & 角点数 \\
\hline 1 & 547 & 472 & 8 & 10 & 8 \\
2 & 7329 & 5448 & 13 & 48 & 0 \\
\hline
\end{tabular}

图 14a 是抽取等值线后的网格结果，每一片都 是一个封闭区域，这是等值线的性质所决定的. 为 保证域之间连续性，会有很少一部分区域出现重 叠. 图 $14 \mathrm{~b}$ 是去除重叠后求取了每一域边界交点, 并划分所有非四边形域的结果. 图 $14 \mathrm{c}$ 是对域边界

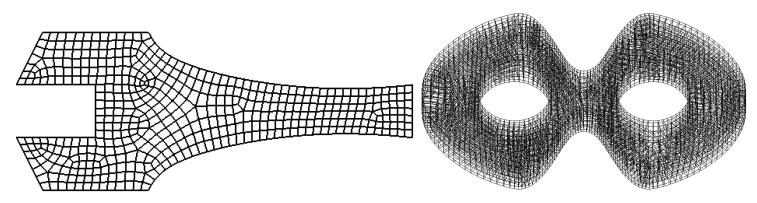

图 13 网格模型

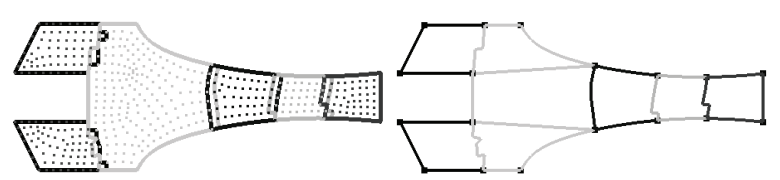

a. 抽取等值网格 b. 去重叠划分四边形域

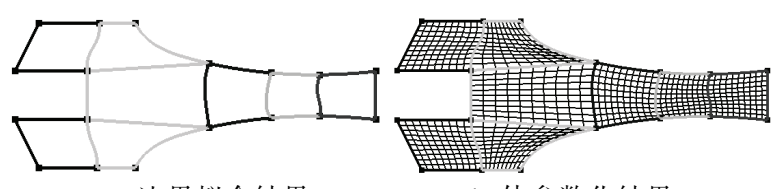

c. 边界拟合结果

图 14 二维模型体参数化 
进行排序, 利用 $\mathrm{B}$ 样条曲线结合最小二乘法反求 边界控制点, 拟合出所有域的边界. 图 $14 \mathrm{~d}$ 是根据 边界控制点插值域内部控制点, 再根据 B 样条体 参数化理论实现参数化.

与图 14 二维模型不同，图 15a 需要将非 8 节 点六面体网格简化为 8 节点六面体网格，8 个节点 一一对应于六面体的 8 个顶点. 其次, 需要提取六 面体模型几何表面网格并对图 15e 中边界求交点 进行邻域对比约束. 图 15f 中子域边界排序算法更 加复杂, 当然三维子域边界排序算法依然适用于 二维几何域。

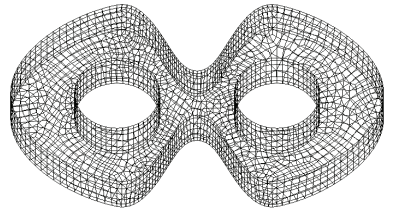

a. 几何表面网格

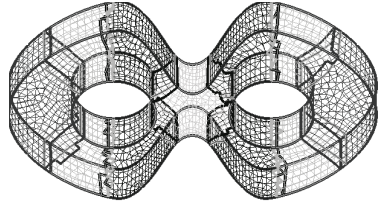

b. 提取等值网格

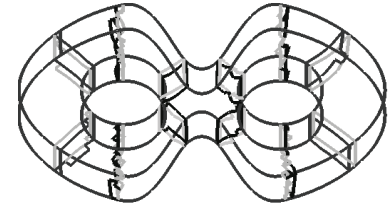

c. 边界重叠现象

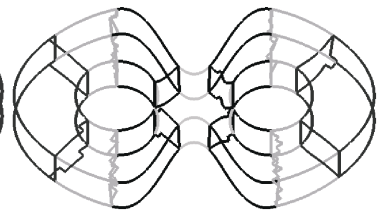

d. 去重叠后结果 e. 子域分割结果

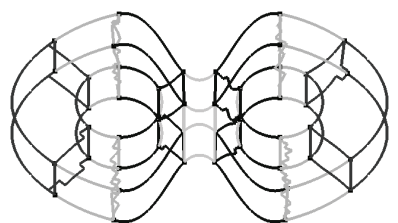

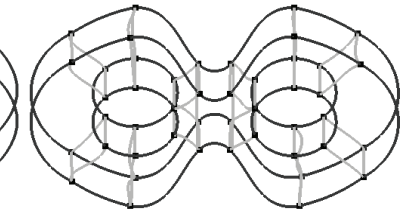

f. 边界拟合结果
图 15 三维模型体参数化

图 16 是二维模型 $\mathrm{B}$ 样条体参数化重构实例. 依次是原始有限元网格模型、等值域提取、边界模 型、去重叠与求交后子域模型、子域边界排序拟合 后模型、 $\mathrm{B}$ 样条体参数化模型. 实例验证了该方法 不仅可以快速、高效地实现多边形有限元模型, 还可 以实现多亏格有限元模型的体参数化重构. 其中, 奇异点主要分布在模型边界上，以保证几何域内 部奇异点数量最少.

三维实例如图 17 所示, 相比有限元模型, 经 过体参数化重建后得到的模型不仅保证了几何表 面的光顺性，减少几何内部的奇异多边形，还使得 内部网格接近于矩形，提高了内部单元质量.

为了表明重建模型能够进行等几何分析，下 面使用扳手模型进行静力学分析验证. 此过程将 涉及多片区域的等几何静力学分析，如图 18 所示. 图 18a 所示为域分割的多片参数化重建模型，对图

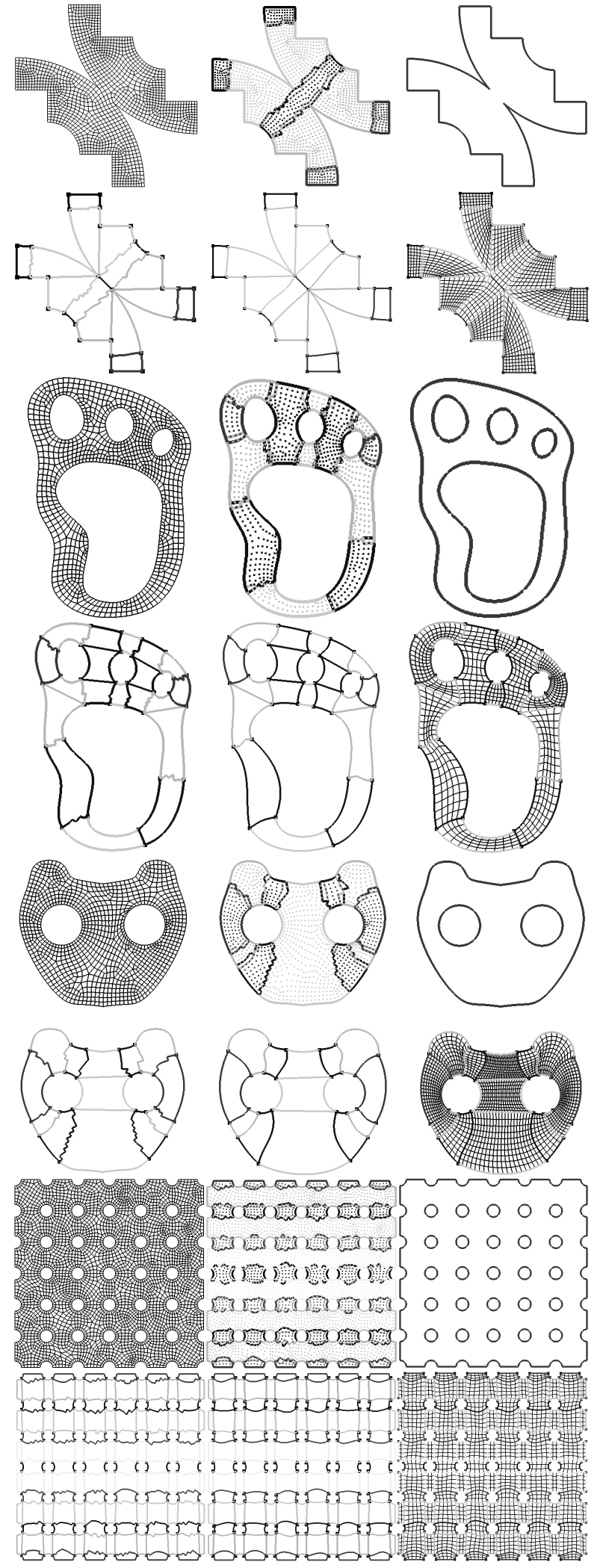

图 16 二维实例重建

$18 \mathrm{~b}$ 所示扳手孔进行全局约束, 在把手处施加向下 的载荷; 等几何分析结果如图 18c 所示, 图 18d 所 示为有限元分析结果对比图. 通过数值对比, 分析 结果误差在 $0.28 \%$ 以内. 

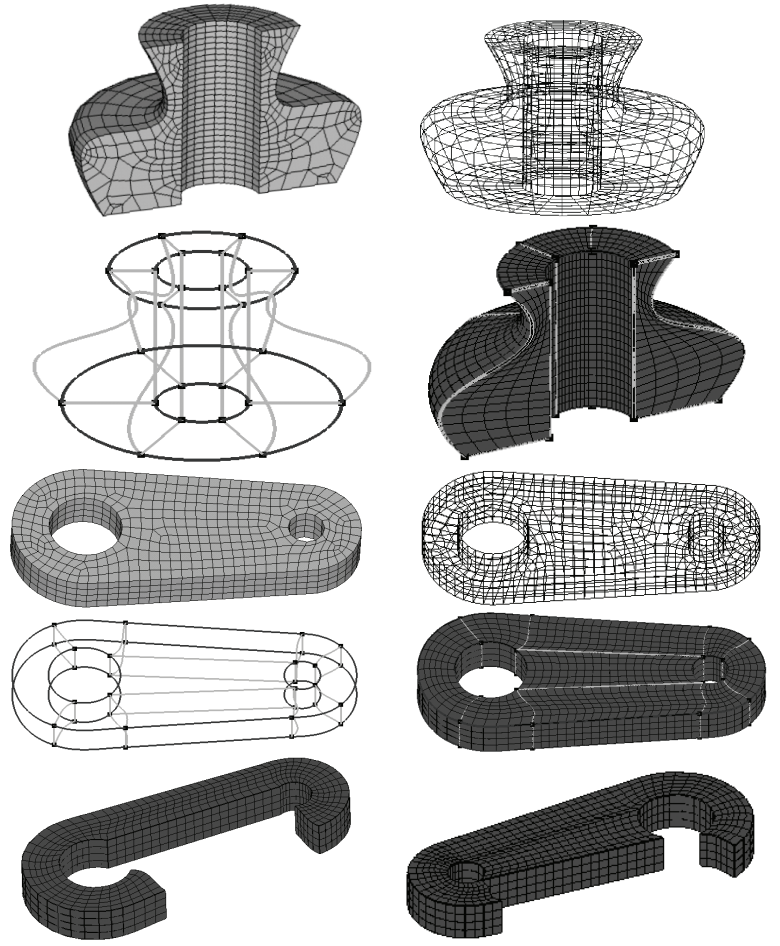

图 17 三维实例重构

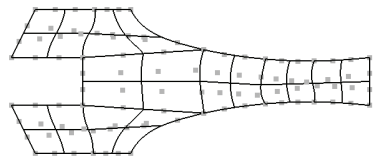

a. 多片参数化模型

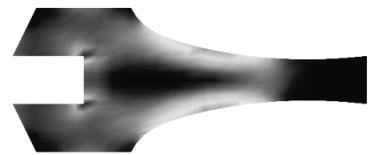

c. 等几何分析结果

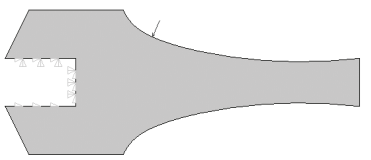

b. 载荷约束

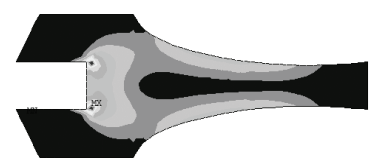

d. 有限元分析结果
图 18 对扳手模型等几何分析

等几何分析在不改变几何模型的边界基础上 进行网格细分, 可在不重新划分网格的情况下进 一步提高分析精度. 传统有限元分析中的网格划 分使得几何边界比 CAD 原模型边界精度低, 即使 采用局部细分的方法也存在不可消除的误差, 因 此在精度上等几何分析比传统有限元分析精度更 高, 分析更便捷.

\section{7 结 语}

本文面向有限元四边形网格或六面体网格进 行体参数化几何重构, 重构模型可直接用于等几 何分析. 针对复杂有限元模型可以快速实现 B 样 条体参数化的目的, 解决以往 B-Rep 与 CSG 无法 表达实体内部的问题, 为非均质材料表达提供模
型支撑. 该方法为拓扑优化后处理提供新思路, 重 建的模型不仅可以用于工业生产制造和优化设计, 也可用于优化分析, 从而使得 $\mathrm{CAD}$ 和 CAE 无缝转 换成为可能. 受制于输人模型的网格剖分方法, 目 前该方法能处理任意复杂的二维有限元四边形网 格模型和较为简单的有限元六面体网格模型. 研 究将零亏格三维实体剖分出适用于体参数化的子 域, 进而将本文方法应用于任意复杂的三维网格 模型, 则是下一步的工作.

\section{参考文献(References):}

[1] Zegard T, Paulino G H. Bridging topology optimization and additive manufacturing[J]. Structural and Multidisciplinary Optimization, 2016, 53(1): 175-192

[2] Liu K, Tovar A. An efficient 3D topology optimization code written in Matlab[J]. Structural and Multidisciplinary Optimization, 2014, 50(6): 1175-1196

[3] Chen Long, Han Wenyu, He Wenbin, et al. The algorithm of slicing and obtaining support points for complex heterogeneous volume parameterization model[J]. Journal of Mechanical Engineering, 2019, 55(15): 99-108(in Chinese)

(陈龙, 韩文瑜, 何文彬, 等. 复杂非均质体参数化模型的切 片及支撑点求解 [J]. 机械工程学报, 2019, 55(15): 99-108)

[4] Liu J K, Gaynor A T, Chen S K, et al. Current and future trends in topology optimization for additive manufacturing[J]. Structural and Multidisciplinary Optimization, 2018, 57(6): 2457-2483

[5] Vogiatzis P, Chen S K, Zhou C. An open source framework for integrated additive manufacturing and level-set-based topology optimization[J]. Journal of Computing and Information Science in Engineering, 2017, 17(4): 1-10

[6] Massarwi F, Elber G. A B-spline based framework for volumetric object modeling[J]. Computer-Aided Design, 2016, 78: 36-47

[7] Mohanadhas B, Govindarajan S K. Modeling the sensitivity of hydrogeological parameters associated with leaching of uranium transport in an unsaturated porous medium[J]. Environmental Engineering Research, 2018, 23(4): 462-473

[8] Li W, Pan Y, Yao Y, et al. Modeling and parametric study of the ultrasonic atomization regeneration of desiccant solution[J]. International Journal of Heat and Mass Transfer, 2018, 127(Pt.A): 687-702

[9] German P, Ragusa J C. Reduced-order modeling of parameterized multi-group diffusion k-eigenvalue problems[J]. Annals of Nuclear Energy, 2019, 134(5): 144-157

[10] Kim B C, Mun D. Feature-based simplification of boundary representation models using sequential iterative volume decomposition[J]. Computers \& Graphics, 2014, 38(2): 97-107

[11] Luo Yuetong, Fan Xiaojing, Yu Shengpeng, et al. Face shell shrinking based B-Rep to CSG conversion algorithm[J]. Journal of Computer-Aided Design \& Computer Graphics, 2014, 26(10): 1673-1680(in Chinese)

(罗月童, 樊晓菁, 俞盛朋, 等. 基于面壳封闭的 B-Rep 至 
CSG 转换算法 [J]. 计算机辅助设计与图形学学报, 2014, 26(10): 1673-1680)

[12] Chen Long, Fan Xingwang, Wang Meng, et al. Parametric reconstruction of four-sided finite element mesh model[J]. Journal of Computer-Aided Design \& Computer Graphics, 2017, 29(4): 680-688(in Chinese)

(陈龙, 樊兴旺, 王猛, 等. 有限元四边单元网格模型的参数 化重建 [J]. 计算机辅助设计与图形学学报, 2017, 29(4): 680-688)

[13] Hu Xianghong, Chen Kangning. Generating quadrilateral meshes by using region growth algorithm[J]. Journal of Computer-Aided Design \& Computer Graphics, 2004, 16(1): 29-34(in Chinese)

(胡向红，陈康宁. 由区域生长算法实现四边形网格划分 [J]. 计算机辅助设计与图形学学报, 2004, 16 (1): 29-34)

[14] Sun Chunhua, Chen Xuefang, Jiang Zuo, et al. Non-self- overlapping structured grid generation by Gregory method[J]. Journal of Computer Applications, 2007, 27(12): 3066-3068(in Chinese)

(孙春华，陈雪芳，姜左，等. 基于 Gregory 法的 $\mathrm{N}$ 边域非自 交结构网格生成[J]. 计算机应用, 2007, 27(12): 3066-3068)

[15] Jian Qun, Lin Hongwei, Cao Qi, et al. Generating quality guaranteed quadrilateral mesh on an $n$-sided region[J]. Journal of Computer-Aided Design \& Computer Graphics, 2016, 28(11): 1811-1820(in Chinese)

(简群, 萄宏伟, 曹琦, 等. 平面 $n$ 边域上高品质四边网格生 成方法 [J]. 计算机辅助设计与图形学学报，2016，28(11): 1811-1820)

[16] Li Yi, Bao Jinsong, Jin Ye, et al. Quadrilateral mesh generation algorithm for planar domain with multi-constraints[J]. Journal of Computer-Aided Design \& Computer Graphics, 2008, 20(4): 488-493(in Chinese)

(李毅, 鲍劲松, 金烨, 等. 二维域多约束四边形有限元网格 生成算法 [J]. 计算机辅助设计与图形学学报, 2008, 20(4): 488-493)

[17] Owen S J, Staten M L, Canann S A, et al. Q-Morph: an indirect approach to advancing front quad meshing $[\mathrm{J}]$.International Journal for Numerical Methods in Engineering, 1999 , 44(9): $1317-1340$

[18] Zhao Guoqun, Yu Song, Wang Guangchun. Automatic generation and regeneration algorithm of hexahedral mesh[J]. Journal of Mechanical Engineering, 2006, 42(3): 188-192(in Chinese) (赵国群，虞松，王广春. 六面体网格自动划分和再划分算 法 [J]. 机械工程学报, 2006, 42 (3): 188-192)

[19] Chen Long, Liu Yusheng, Xu Gang. Generation of volume parameterization model based on discrete harmonic mapping $[\mathrm{J}]$. Journal of Image and Graphics, 2015, 20(4): 568-575(in Chinese) (陈龙, 刘玉生, 徐岗. 体参数化模型离散调和映射生成 $[\mathrm{J}]$.
中国图象图形学报, 2015, 20(4): 568-575)

[20] Al Akhras H, Elguedj T, Gravouil A, Rochette M. Isogeometric analysis-suitable trivariate NURBS models from standard B-Rep models[J]. Computer Methods in Applied Mechanics and Engineering, 2016, 307(8): 256-274

[21] Martin T, Cohen E, Kirby R M. Volumetric parameterization and trivariate B-spline fitting using harmonic functions $[\mathrm{J}]$. Computer Aided Geometric Design, 2009, 26(6): 648-664

[22] Aigner M, Heinrich C, Júttler B, et al. Swept volume parameterization for isogeometric analysis[C]//Proceedings of the 13th IMA International Conference on Mathematics of Surfaces XIII. Heidelberg: Springer, 2009: 19-44

[23] Xu G, Mourrain B, Wu X Y, et al. Efficient construction of multi-block volumetric spline parameterization by discrete mask method[J]. Journal of Computational and Applied Mathematics, 2015, 290(15): 589-597

[24] Tarini M, Puppo E, Panozzo D, et al. Simple quad domains for field aligned mesh parametrization[J]. ACM Transactions on Graphics, 2011, 30(6): Article No.142

[25] Kowalski N, Ledoux F, Frey P. Automatic domain partitioning for quadrilateral meshing with line constraints $[\mathrm{J}]$. Engineering with Computers, 2015, 31(3): 405-421

[26] Li Tianhua. Research on quad remeshing based on vector field[D]. Changchun Jilin University. College of Computer Science and technology, 2017(in Chinese)

(李天华. 基于方向场的四边形重网格化技术研究[D]. 长春: 吉林大学计算机科学与技术学院, 2017)

[27] Feng S, Leung A K, Liu H W, et al. Effects of thermal boundary condition on methane oxidation in landfill cover soil at different ambient temperatures[J]. Science of the Total Environment, 2019, 692(7): 490-502

[28] He Bingpeng, Feng Renzhong, Yu Shengjiao. B-spline curve and surface fitting using differential evolution algorithm[J]. Journal of Graphics, 2016, 37(2): 178-183(in Chinese) (何兵朋, 冯仁忠, 余胜蛟. 基于差分进化算法的 $\mathrm{B}$ 样条曲线 曲面拟合[J]. 图学学报, 2016, 37(2): 178-183)

[29] Zhao X Y, Zhang C M, Yang B, et al. Adaptive knot placement using a GMM-based continuous optimization algorithm in B-spline curve approximation[J]. Computer-Aided Design, 2011, 43(6): 598-604

[30] Feng Renzhong, Zha Li. Local approach to construct cubic B-spline interpolation curve and surface with $C^{2}$ continuity[J]. Journal of Engineering Graphics, 2005, 26(6): 110-117(in Chinese)

(冯仁忠, 查理. 局部构造 $C^{2}$ 连续的三次 $\mathrm{B}$ 样条插值曲线和 双三次插值曲面 [J]. 工程图学学报, 2005, 26(6): 110-117)

[31] Hao Y X, Li C J. The $C^{1}$ and $C^{2}$ quasi-plateau problems[J]. Journal of Computational and Applied Mathematics, 2018, 329(5): 106-124 